\title{
EXTINCTION AND REIGNITION IN COUNTERFLOW SPRAY DIFFUSION FLAMES INTERACTING WITH LAMINAR VORTICES
}

\author{
VITO S. SANTORO AND ALESSANDRO GOMEZ \\ Yale Center for Combustion Studies \\ Department of Mechanical Engineering \\ Yale University \\ New Haven, CT 06520-8286, USA
}

\begin{abstract}
The interaction of laminar vortices with a methanol spray counterflow diffusion flame was studied experimentally with vortices generated from either the fuel side or the oxidizer side. The overall stoichiometry was such that the flame resided on the fuel side of the gas-stagnation plane. Local extinction and subsequent reignition were investigated as the circulation of the vortex was varied. It was found that extinction required vortices of larger circulation if generated from the oxidizer side. The effect was attributed to stretching, and possibly, partial dissipation of the vortex, as it approached the stagnation plane before interacting with the flame. The robustness of the spray flame to vortex-induced extinction was compared with that of a similar gaseous flame. The spray flame was found to be comparatively weaker. Of the two potential culprits for such a difference, namely, the energetic handicap of spray flames due to the latent heat of vaporization of the liquid fuel and droplet inertia, the former was found to be the dominant factor. After extinction occurred, a hole was created in the diffusion flame, confining the combustion process to an annular region. The flame was then able to propagate back toward the centerline, re-establishing itself as a flat diffusion flame. The time interval for this reignition process was investigated as a function of the vortex circulation. It was found that, if the vortex approached the flame from the fuel side, the reignition time was much shorter than when the vortex was injected from the oxidizer side, and decreased for increasing values of the vortex circulation. In contrast, the reignition time increased with the circulation, if the vortex approached the flame from the oxidizer side. Only in the latter case, droplet inertia played a role in the reignition process.
\end{abstract}

\section{Introduction}

The interaction of laminar vortices with a flat flame offers a scenario in which curvature and timedependent effects can be investigated in a controlled fashion. The results obtained from this idealized configuration are useful to understand fundamental aspects of laminar flames and can be used to enhance turbulent modeling based on the flamelet approach [1]. A review of the recent progress made on this subject was presented in Ref. [2]. With respect to curvature effects, it was shown numerically that in hydrogen flames, modest curvatures, combined with preferential diffusion, produce dramatic effects on the flame structure and extinction conditions $[3,4]$. The possibility of annular extinction was demonstrated in Ref. [3], while the value of the scalar dissipation rate at extinction for hydrogen flames was found to vary dramatically, depending on whether the vortex approached from the fuel side or the oxidizer side, in Ref. [4]. Time-dependent effects were investigated in a study in which it was shown that diffusion flames perturbed by a vortex sustain much higher extinction strain rates than quasi-steady flames [5]. Furthermore, it was shown theoretically that the scalar dissipation rate is a variable better suited to characterize extinction, being invariant with the mode (either steady or unsteady) of extinction. This result was at first corroborated experimentally by using an approximation for the scalar dissipation rate in terms of the thickness of the mixing layer. Subsequently, it was confirmed within the experimental error of the technique by detailed measurements of the scalar dissipation rate by Raman spectroscopy [6]. The effect of the timescale of the vortex on relatively slow chemical kinetic processes, such as the production of nitric oxide, was investigated in Ref. [7]. The peak mass fraction of NO was demonstrated to vary with the timescale of the perturbation, and the effects of the vortex on the different formation paths were sorted out computationally.

Although gaseous flames are a convenient starting point and have been instrumental to shed light on relevant features of diffusion flames, most of the practical non-premixed combustion originates from liquid fuel in some form of turbulent spray flame. Surprisingly, to date, little has been done in the area of spray-flame vortex interaction. An isolated theoretical work was presented in Ref. [8], and a numerical study of a cluster of droplets burning in the 


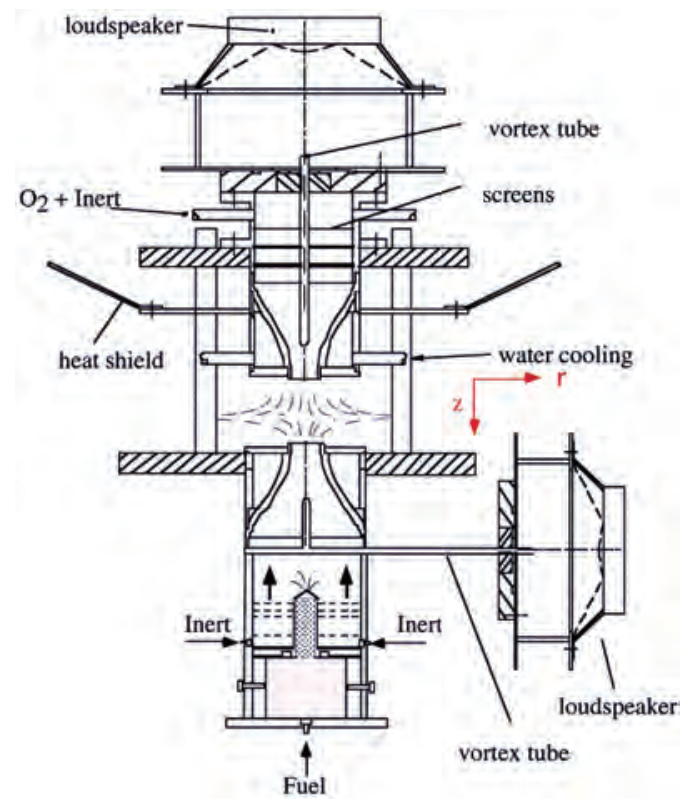

FIG. 1. Burner configuration.

core of a vortex was presented in Ref. [9]. The behavior of laminar droplets in counterflow configurations and in a vortex pair was analyzed numerically for cold conditions in Ref. [10]. Aggarwal et al. [11] investigated the effects of vortical structures on droplets dispersion in a jet-spray flame. The only experimental work on the interaction of vortices and laminar spray flames was presented in Ref. [12]. It was shown that, when the vortex was introduced from the oxidizer side, the spray flame had similar features to those of a gaseous flame. Namely, the vortex first extinguished the flame at a strain rate higher than the quasi-steady extinction value, and then the flame healed itself. Most importantly, it was shown for a specific flame that the time necessary for flame to re-establish itself after being locally quenched was significantly higher for the spray flame than for the gaseous counterpart. This result was explained in terms of the inertia of the droplets centrifuged off the centerline by the incoming vortex from the opposite direction, which resulted in momentary fuel depletion along the centerline and a delay in reignition.

This work builds on the results of Ref. [12] and systematically examines experimentally the extinction of spray methanol flames, as induced by vortices introduced from the fuel or the oxidizer side. As will be shown, the differences in the phenomenology of these two cases are both quantitative and qualitative. The primary reason is that, since the gas-stagnation plane is displaced with respect to the flame, only the vortex originating from one of the two sides interacts with this stagnation region before affecting the flame. Extinction conditions are characterized based on the circulation of the vortices injected in the system and compared with the circulation required to extinguish identical flames in which the fuel had been prevaporized. Last, the time interval during which combustion is quenched by the vortex is measured as a function of the circulation of the vortex and compared in both sprays and gaseous flames.

\section{Experimental Methods}

An axisymmetric counterflow diffusion flame was established in a vertical configuration, as shown in Fig. 1, with the oxygen and nitrogen being fed from one side, methanol and nitrogen from the other. A commercial ultrasonic nebulizer was used to disperse the liquid fuel. This device atomizes modest liquid flow rates, imparting a small velocity to the liquid drops. The typical droplet-size distribution obtained with this device was presented in Ref. [13] and applies for the experimental conditions described below. Both sides of the burner terminated in a contraction to optimize the uniformity of the axial velocity in the radial direction. The exit diameter of the two nozzles was $12.5 \mathrm{~mm}$, and the separation distance between them was kept constant at $13.5 \mathrm{~mm}$. Provision was made to recover and measure the liquid loss, to measure precisely the fuel flow rate entering the combustion region, as in Ref. [14].

The steady flames were perturbed by laminar vortices generated periodically from either the oxidizer or the fuel side. The vortex generation scheme for the oxidizer side was described in Ref. [5]. The one adopted on the fuel side, instead, was more elaborate because of the special requirements of the twophase stream. A loudspeaker positioned horizontally with respect to the burner axis was connected to a horizontal tube cutting through the lower burner. Half-way into the burner, a separate tube was soldered on the horizontal one and positioned in coincidence with the burner axis. The end of the horizontal tube opposite to the loudspeaker was sealed. The whole system was mounted sufficiently downstream of the nebulizer to minimize any effect on the atomization of the liquid fuel. Suitably synthesized voltage functions were applied to the loudspeaker on either the fuel side or the oxidizer side, forcing air impulsively through the $1.5 \mathrm{~mm}$ tube. As a result, a puff of gas was released by either tube and rolled up into an axisymmetric toroidal vortex coaxial with the burner. The amplitude of the voltage function was used to control the strength of the vortex.

The vortices were visualized using planar light scattering of $1.5-\mu \mathrm{m} \mathrm{Al}_{2} \mathrm{O}_{3}$ particles using the third harmonic of a Nd:YAG laser. The flame dynamics were monitored using planar-induced fluorescence 


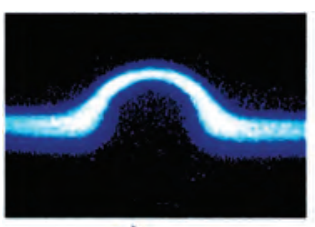

a)

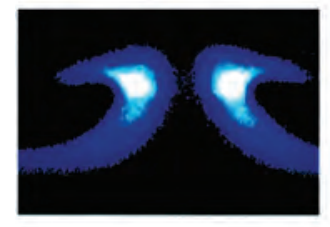

c)

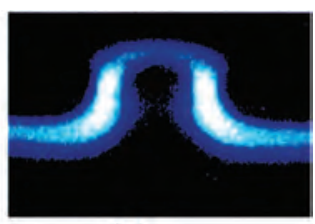

b )

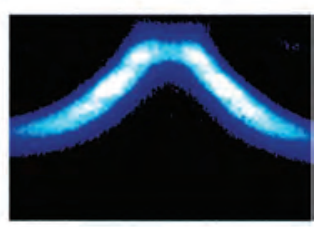

d)
FIG. 2. Sequence of formaldehyde images at $t=3 \mathrm{~ms}$ (a), $t=4 \mathrm{~ms}$ (b), $t=6 \mathrm{~ms}$ (c), and $t=10 \mathrm{~ms}$ (d) for a vortex approaching the flame from the fuel side. The size of the image in the horizontal direction corresponds to $12 \mathrm{~mm}$.

of formaldehyde. As shown in Ref. [12], formaldehyde is an ideal complementary marker of methanol flames as an intermediate in the dominant oxidation path of methanol: $\mathrm{CH}_{3} \mathrm{OH} \rightarrow \mathrm{CH}_{2} \mathrm{OH} \rightarrow \mathrm{CH}_{2} \mathrm{O} \rightarrow$ $\mathrm{HCO} \rightarrow \mathrm{CO}$. Its consumption generates formyl radical, $\mathrm{CHO}$, that tracks very well the position of the peak of heat release, as one-dimensional unsteady numerical calculations for a gaseous flame with detailed chemistry and transport properties showed [6]. Consequently, as previously shown in Ref. [12], the consumption of formaldehyde coincides spatially with the peak of the heat release, and the formaldehyde layer is expected to be adjacent to the region of peak of heat release. Moreover, the pyrolysis of $\mathrm{CH}_{2} \mathrm{OH}$ to $\mathrm{CH}_{2} \mathrm{O}$ was shown to be the critical step for extinction of methanol flames [15].

Similarly to Refs. $[5,12]$, the third harmonic of a $\mathrm{Nd}$ :YAG laser, at $355 \mathrm{~nm}$ and with $120 \mathrm{~mJ} /$ pulse of energy, was used to excite the $4_{0}^{1}$ transition in the $\tilde{A}^{1} A_{2} \leftarrow \tilde{X}^{1} A$ band of $\mathrm{CH}_{2} \mathrm{O}$. The resulting signal was detected using a gated single-stage image intensifier coupled to a CCD (Santa Barbara Instrument Group ST6B). A narrow interference filter at $415 \mathrm{~nm}$ with a half-width of $7 \mathrm{~nm}$ was used to reject flame luminescence and other interferences. Because of the spray diluteness, droplet Mie scattering did not require additional filtering. This diagnostic technique and the vortex visualization were synchronized, using a variable time delay, with the same signal generator that produced the vortices. In this way, the evolution of the interaction between the vortex and flame could be monitored at any desired instant in time.

The gas velocity, the velocity and size distribution of the droplets, and, ultimately, the vortex circulation were measured using a commercial phase Doppler Anemometer (PDA) (Dantec) [13]. In particular, the velocity of the gas on the oxidizer side was determined using seed particles of $\mathrm{Al}_{2} \mathrm{O}_{3}$, with a nominal diameter of $1.5 \mu \mathrm{m}$. The velocity of the gas on the fuel side, instead, was determined using droplets with diameter smaller than $8 \mu \mathrm{m}$, which had estimated Stokes number smaller than 0.1. The singlepoint velocity measurements were phase-averaged, using the voltage function that generates the vortices.

Results are present for a methanol flame with the following boundary conditions: $v_{\mathrm{ox}}=0.65 \mathrm{~m} / \mathrm{s}$, $v_{\mathrm{f}}=0.56 \mathrm{~m} / \mathrm{s}, Y_{\mathrm{f}}=0.20$, and $Y_{\mathrm{ox}}=0.35$, where $v$ is the axial velocity along the centerline, $Y$ is the mass fraction, and the subscripts ox and $f$ refer to oxidizer side and fuel side, respectively. Flame stabilization required $10 \%$ of the total fuel to be prevaporized before been inserted into the burner. The prevaporized fuel alone was not sufficient to establish a flame. Thus, the phenomenology to be described is indeed typical of a laminar spray flames.

\section{Results and Discussion}

A flat flame was established between the two nozzles and perturbed by vortices generated from either the fuel side or the oxidizer side. A sequence of the interaction of the methanol flame with a vortex generated from the fuel side is presented in Fig. 2. The vortex curved the flame $3 \mathrm{~ms}$ after it had been generated, as the formaldehyde layer shows in Fig. 2a, and extinguished it at the centerline at $t=5 \mathrm{~ms}$, as indicated by the even fainter fluorescence in that region in Fig. 2b. After the flame opened up momentarily (Fig. 2c at $t=6 \mathrm{~ms}$ ), it then closed in again on the centerline at $t=10 \mathrm{~ms}$. Fig. 3 visualizes three different aspects of the interaction at $t=6 \mathrm{~ms}$, corresponding to Fig. 2c. The top snapshot presents planar light scattering off the seeded alumina particles in white color, with a clear identification of the position of the vortex at that time; in the middle one, a juxtaposition of the vortex and the formaldehyde layer in blue is presented, with a clear identification of how the vortex penetrated and disrupted locally the flame. Finally, the last photograph was obtained without seed particles by imaging the scattering off the droplets that all appear to be located near the axis and inside the vortex, but not in the roll-up region, presumably because they had been centrifuged off by inertia effects. It is clear from this image that, after the vortex has "punched through" the flame, the flow configuration is such that the vortex rollup induces a radial velocity that, after the local flame strain has relaxed to a value at which combustion can be re-established, helps the edge flame to move toward the centerline and bring about healing of the flame. 

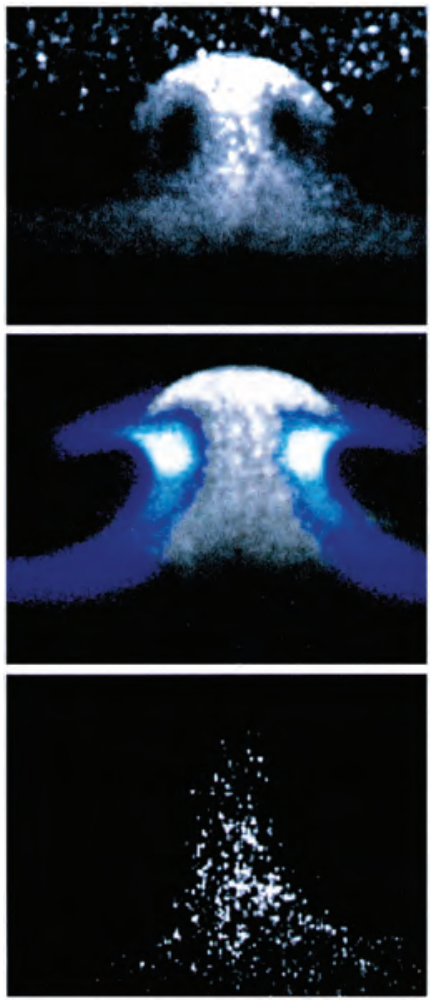

FIG. 3. Image at $t=6 \mathrm{~ms}$ of the vortex (a), of the vortex plus formaldehyde (b), and of the droplets for a vortex approaching the flame from the fuel side (c). The size in the horizontal direction corresponds to $12 \mathrm{~mm}$.

The corresponding sequence for the case of the vortex approaching the flame from the oxidizer side is shown in Figs. 4 and 5. The formaldehyde sequence in Fig. 4 highlights a similar phenomenology to that discussed in Fig. 3, but with two significant differences: first, the flame is far less curved than when the vortex had been injected from the fuel side; second, the sequence is further "stretched" in time, with flame curving appearing $3 \mathrm{~ms}$ after vortex generation (Fig. 4b), extinction occurring at $t=$ $6 \mathrm{~ms}$ (Fig. 4c), and the flame healing itself at $t=$ $28 \mathrm{~ms}$ (Fig. 4d). The first observation can be explained by the fact that the vortex traveling from the oxidizer side is stretched as it approaches the gasstagnation plane, before it interacts with the flame. During this process, its size increases considerably, and, consequently, so does the radius of curvature induced on the flame. Fig. 5 shows, in the top photograph, obtained by feeding particles from both sides of the burner, the visualization of the stretched vortex, as it begins to penetrate the fuel side. Superimposed on the particle scattering is the fluorescence image in blue from the formaldehyde layer in

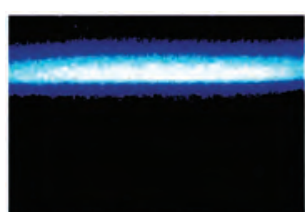

a)

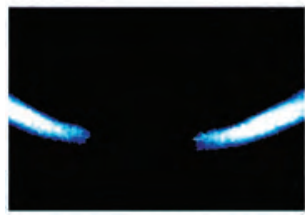

c)

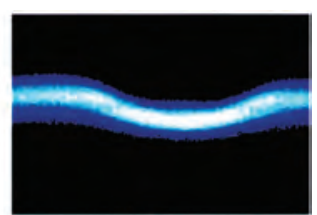

b)

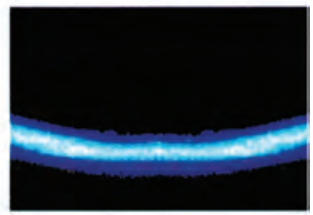

d)
FIG. 4. Sequence of formaldehyde images at $t=0 \mathrm{~ms}$ (a), $t=3 \mathrm{~ms}$ (b), $t=6 \mathrm{~ms}$ (c), and $t=28 \mathrm{~ms}$ (d) for a vortex approaching the flame from the oxidizer side. The size of the image in the horizontal direction corresponds to $12 \mathrm{~mm}$.

the middle picture. The last image was obtained by scattering the pulsed laser sheet off the droplets only. All three photographs were obtained at $t=$ $8 \mathrm{~ms}$ after vortex generation. Contrary to the case discussed in Fig. 3, the flow induced by the vortex after extinction opposes the propagation of the edge flames toward the centerline [16], as can be inferred from the position of the stretched vortex cores.

Extinction induced by a vortex can be quantified by measuring the intensity of the perturbation in terms of vortex "strength" characterized by the circulation of the vortices, as measured at the nozzle exit before the vortex interacts with the combustion region. This, in some sense, corresponds to conditions at "infinity" in the interaction of a flamelet with a vortex in a practical system. The circulation is defined as follows:

$$
\Gamma=\oint \mathbf{u} \cdot \mathrm{d} \mathbf{l}=\sum_{i} \int u_{i} \mathrm{~d} l_{i}
$$

where $\mathbf{u}$ is the velocity vector. A rectangular path was chosen for convenience, with one side along the centerline, two sides along the radial direction, and the last side parallel to the $z$-axis. Therefore, the integral reduces to:

$$
\begin{aligned}
\Gamma= & \int_{r=0} v_{\mathrm{z}} \mathrm{d} z+\int_{z=0} v_{r} \mathrm{~d} r \\
& +\int_{r=\mathrm{r}^{*}} v_{z} \mathrm{~d} z+\int_{z=\mathrm{z}^{*}} v_{r} \mathrm{~d} r
\end{aligned}
$$

The path was chosen so that it would contain half of the toroidal vortex, and the minimal dimensions of this rectangle were established based on vortex 

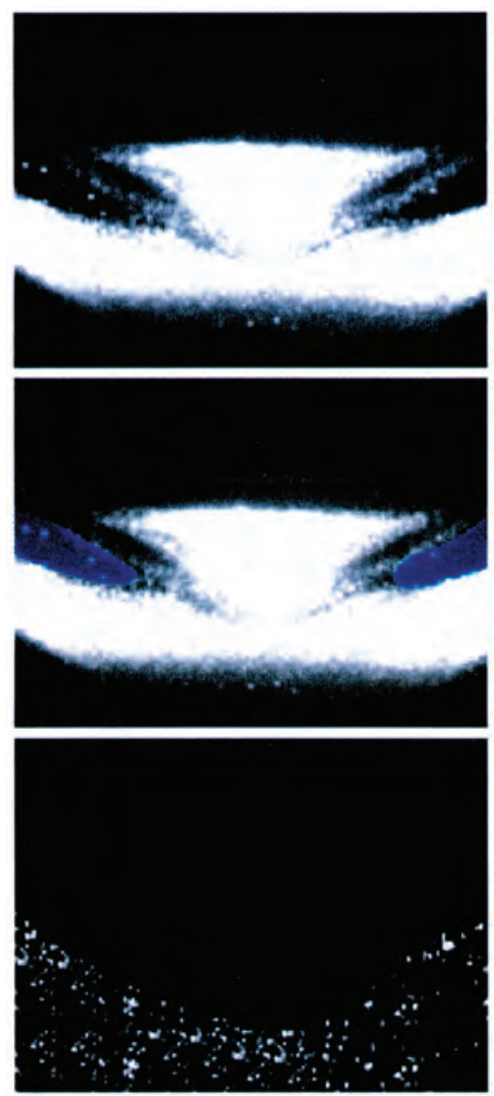

FIG. 5. Image at $t=6 \mathrm{~ms}$ of the vortex (a), of the vortex plus formaldehyde (b), and of the droplets for a vortex approaching the flame from the oxidizer side (c). The overall size in the horizontal direction corresponds to $16 \mathrm{~mm}$.

visualization. The velocity profiles along the chosen rectangular path were measured as a function of time using the PDA. The voltage function that generates the vortices was used as an external trigger for the instrument to mark an absolute reference time, similarly to the other visualization techniques used. To obtain the velocity of the vortex, the steady-velocity components had to be subtracted from the measurements. Using the external reference time, the temporal velocity measurements at the various locations were converted to a velocity field map at a fixed time, as in Ref. [5]. Thereafter, the integral could be evaluated at any arbitrary time. For the circulation of the vortex to be meaningful, the integral needed to be calculated only when the vortex is fully enclosed in the integration path. This time was chosen by using vortex visualization.

We shall now examine the threshold circulation beyond which flame extinction is induced. The comparison will first focus on the same spray flame, con- trasting vortex injection from the fuel side with that from the oxidizer side. In the first case, extinction was achieved when the circulation of the vortex exceeded $12 \mathrm{~cm}^{2} / \mathrm{s}$; in the second, a minimum value of $30 \mathrm{~cm}^{2} / \mathrm{s}$ was needed. The difference in vortex strength can be explained by recalling that the vortex generated from the oxidizer side needs to displace the gas-stagnation plane before reaching the flame and extinguishing it, as shown in Fig. 5. Equation 2 can be approximated as $\Gamma=\bar{v} \oint \mathrm{d} l$. As the gas-stagnation region stretches the vortex, the line integral increases, which, for constant circulation, would yield a decrease of the average velocity. As a result, the vortex translational velocity that momentarily increases the flame-strain rate, is reduced and its effect on the flame is attenuated. The attenuation is even more significant if one relaxes the hypothesis of constant circulation and considers the dissipation of the vortex in the viscous layer near the stagnation region.

Similar measurements can also be used to contrast this spray flame with a purely gaseous one in which the fuel was prevaporized before being introduced into the burner. The circulation of the vortex that extinguished this gaseous flame was measured at 25 and $52 \mathrm{~cm}^{2} / \mathrm{s}$, depending on whether the vortex had been injected from the fuel side or the oxidizer side. These results show that spray flames are inherently weaker than gaseous ones, since they extinguish at lower vortex strengths. These results are consistent with the findings in Ref. [17], in which vortex-induced extinction was characterized in terms of the local strain rate at the flame. In all of these spray flames, the droplets, with mean diameter of $25 \mu \mathrm{m}$ and small slip with respect to the gas phase, vaporized before reaching the flame, even for very high values of the vortex circulation. These kinds of flames were labeled pseudogaseous in Ref. [18]. The inherent relative weakness of spray flames was predicted theoretically in Refs. [19-21] and demonstrated experimentally in Ref. [19] for quasi-steady extinction. The theoretical work showed that spray flames have lower temperatures than gaseous ones because part of the energy of combustion is used to vaporize the fuel droplets. A critical parameter, the ratio of the heat of vaporization to the heat of combustion of the liquid fuel was identified. As such a ratio increases, the heat loss on the fuel side of the flame increases and so does the extinction Damköhler number. In contrast, under conditions of direct droplet/flame interaction, if droplets with large slip and initially large diameter penetrate through the flame, as observed only in Ref. [22], unique conditions in which spray flames appear to be reinvigorated by the presence of the droplets are even possible, resulting in the observation of higher peak temperatures under conditions far from extinction.

In the case of flame/vortex interaction, droplet inertia can potentially modify the local concentration of fuel at the flame, influencing the extinction 


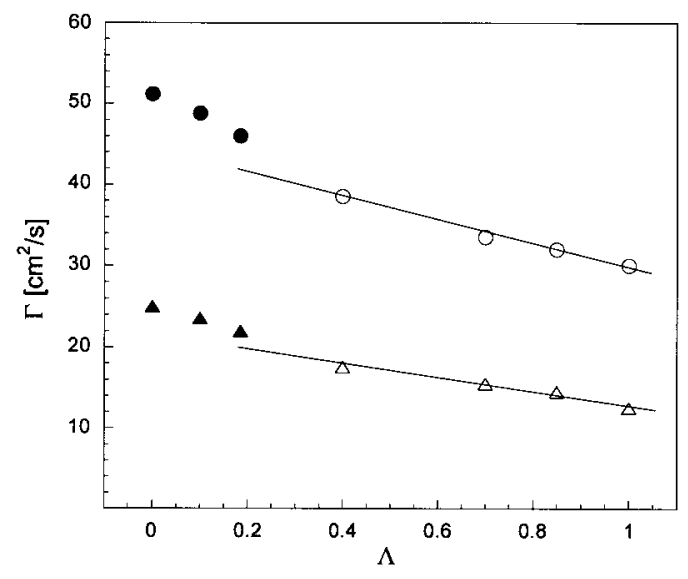

FIG. 6. Circulation of the vortices that cause extinction as a function of the non-dimensional energy per unit time 1. The extinction conditions are shown for a vortex approaching the flame from the oxidizer side (circles), and for the vortex approaching the flame from the fuel side (triangles). Solid symbols are used for gaseous flames, and open symbols for spray flames.

conditions. It is therefore interesting to discriminate between energetic effects and inertia effects systematically. To that end, the latent heat of vaporization of the liquid fuel was compensated by changing the thermal energy input into the system. Specifically, the temperature of the gas on the oxidizer side can be modified by keeping the temperature of the fuel side unchanged, thus leaving the droplet-size distribution unaffected. A non-dimensional energy parameter $\Lambda$, defined as

$$
\Lambda=\frac{\dot{m}_{\mathrm{l}} h_{\mathrm{v}}-c_{\mathrm{p}} \dot{m}_{\mathrm{ox}}\left(T_{\mathrm{ox}}-T_{\mathrm{xo}, 0}\right)}{\dot{m}_{\mathrm{ls}} h_{\mathrm{v}}}
$$

with $\dot{m}_{1}$ the fuel mass flow rate introduced as liquid droplets into the burner, $\dot{m}_{\mathrm{ox}}$ and $T_{\mathrm{ox}}$ the mass flow rate and temperature of the gas introduced from the oxidizer side, $T_{\mathrm{ox}, 0}=300 \mathrm{~K}$ as reference temperature, $\dot{m}_{\mathrm{ls}}$ the fuel mass flow rate for a purely spray flame, and $h_{\mathrm{v}}=37.43 \mathrm{~kJ} / \mathrm{mol}$. This parameter was constructed to be equal to zero for a gaseous flame with the oxidizer introduced at $T_{\mathrm{ox}}=T_{\mathrm{ox}, 0}$ and equal to one for a spray flame with $T_{\mathrm{ox}}=T_{\mathrm{ox}, 0}$. Intermediate points can be obtained by either increasing $T_{\text {ox }}$ in the spray flame or decreasing it in the gaseous flame, which is simpler than using mixtures of vapor and spray, the mass flow rates of which are difficult to quantify. The threshold circulation of the vortices that cause extinction is presented in Fig. 6 as a function of the non-dimensional energy parameter $\Lambda$. The upper data set was obtained for vortex injections from the oxidizer side, the lower one with injection from the fuel side. The two outermost points in each set correspond to purely gaseous flame $(\Lambda=0)$ and purely spray flame $(\Lambda=1)$, both with $T_{\mathrm{ox}}=\mathrm{T}_{\mathrm{ox}, 0}$. Intermediate points were obtained by either increasing the temperature on the oxidizer side of the spray flame from 300 to $363 \mathrm{~K}$, for $0.4 \leq \Lambda \leq 1$, or decreasing such temperature in gaseous flames from 300 to $278 \mathrm{~K}$, corresponding to $0 \leq \Lambda \leq 0.2$. The flange on the oxidizer side was water cooled by a thermostatic bath to control the temperature of the inlet gas accurately (Fig. 1). Unfortunately, this temperature could not be increased beyond $T_{\mathrm{ox}}=363 \mathrm{~K}$ to avoid structural damage to the burner, whereas, at the prevailing flow rates, an increase of $125 \mathrm{~K}$ would have been necessary to bring about a situation energetically equivalent to the purely vapor flame. Nor was a decrease below $278 \mathrm{~K}$ possible, to avoid condensation of water vapor on the upper flange of the burner and subsequent dripping into the flame. These limitations prevented us from making a comparison at exactly the same value of the energy parameter $\Lambda$. Nevertheless, the experimental results show that heat of vaporization effects account for most of the discrepancy between the circulation at extinction of spray flames and gaseous flames. In fact, we observe that if we linearly extrapolate the value of the extinction circulation for spray flames (four rightmost points) to $\Lambda=0.2$, the difference between spray flames and gaseous flames is relative small, as compared with the difference between pure gaseous $(\Lambda=0)$ and purely spray flames $(\Lambda=$ 1). Probably inertia effects account for this small difference and, as a result, are likely to be of secondary importance even under vortex-flame interaction conditions. Similar results to the ones presented in Fig. 6 were obtained for flames generated by burning liquid heptane as a fuel and are not reported here for space limitations.

As discussed in connection with Figs. 2 and 4, the time required for the flame to reignite after extinction was found to change dramatically, depending on whether the vortex was injected from the fuel side or the oxidizer side occurs was investigated in more details. In Fig. 7, this reignition time is presented as a function of the vortex circulation for both purely gaseous flames and purely spray ones. As for Fig. 6, circles are used for injection from the oxidizer side and triangles for injection from the fuel side, with full symbols pertaining gaseous flames. The leftmost points in each data set represent the extinction conditions for the four different cases examined. The time of reignition for a case in which the vortex is generated on the fuel side decreases for higher values of the circulation for both gaseous and spray flames. An explanation for these results can be based on Fig. 3. The vortex cores create a radial velocity that pushes the flame toward the centerline. This radial velocity increases with the circulation of the vortex, decreasing, as a consequence, the time of 


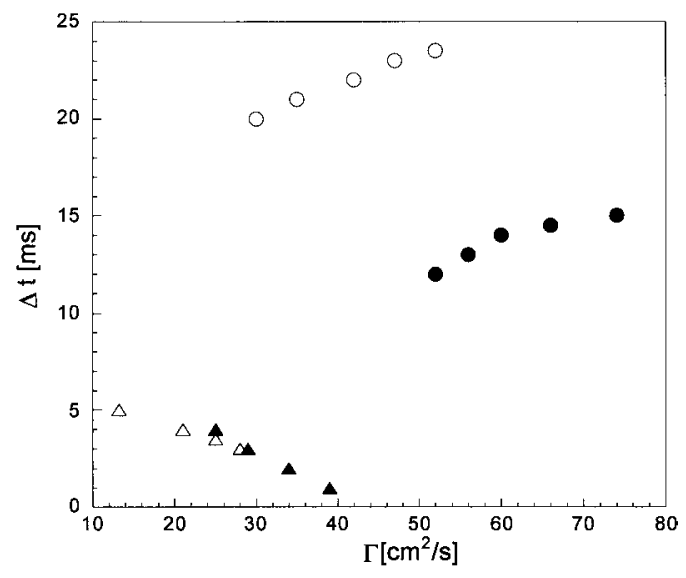

Fig. 7. Time of reignition for spray flames (solid symbols) and gaseous flames (open symbols) in the case of vortices approaching the flame from the fuel side (triangles) and oxidizer side (circles) as a function of the vortex circulation.

reignition. In the case of the vortex generated from the oxidizer side, the time of reignition increases with the circulation. This can be explained by considering Fig. 5. The edge flame in this configuration encounters a negative radial velocity created by the vortex that tends to oppose its propagation toward the center. As a result, the reignition time in this configuration is larger than the case of a vortex approaching from the fuel side, as Fig. 7 shows. Moreover, for larger values of the vortex circulation, the radial velocity created by the vortex increases, increasing, as a consequence, the time required for the flame to re-establish itself. For the same boundary conditions, if we now compare reignition time between spray flames and gaseous ones, we observe that if the vortex approaches the flame from the fuel side, no major differences in reignition time exist. On the other hand the difference is significant if the vortex approaches the flame from the oxidizer side, as already observed in Ref. [12] for one particular flame. This difference was attributed to the vortex displacing the droplets from the centerline, and as a result, preventing the fuel from reaching the region in which extinction had occurred. Fig. 5 visually confirms this explanation. As time progresses, the vortex travels further down on the fuel side reducing the amount of droplets along the centerline even more. Fig. 3, on the contrary, shows that when the vortex is injected from the fuel side, the droplets are always present in the region in which extinction has occurred and the depletion effect is absent. The availability of fuel in this region allows the flame to reignite in a time interval comparable with gaseous flames.

\section{Conclusions}

The interaction of a counterflow methanol spraydiffusion flame with laminar vortices, generated from either the fuel side or the oxidizer side, was investigated experimentally. The extinction conditions were characterized based on the vortex circulation measured at the inlet of the burner. It was shown that the initial circulation of the vortices that caused extinction was larger if the vortex approached the flame from the oxidizer side. The relatively weaker impact of the vortex on the flame was caused by the stretching of the vortex approaching the stagnation plane. Similar results were obtained for a flame in which the liquid fuel was vaporized before being introduced in the burner. Comparing these two sets of data, the spray flame was shown to be invariably weaker than the gaseous one. Of the two potential reasons that could account for this phenomenon, namely, the latent heat of vaporization of the liquid fuel and droplets inertia, the former was shown to be the most important. After extinction occurred and a hole was created in region close to the centerline, the flame propagated back toward the centerline, re-establishing its originally flat shape. The time interval for this reignition process was investigated as a function of the vortex circulation. It was found that, if the vortex approached the flame from the fuel side, the reignition time was much shorter and decreased for increasing values of the vortex circulation. In contrast, the reignition time increased with the circulation, if the vortex approached the flame from the oxidizer side. In the latter case, droplet inertia played a significant role on the reignition process.

\section{Acknowledgments}

The support of NSF grant CTS-9904296 (Dr. Farley Fisher, Contract Monitor) is gratefully acknowledged.

\section{REFERENCES}

1. Peters, N., Prog. Energy Combust. Sci. 10:319 (1984).

2. Renard, P. H., Thévenin, D., Rolon, J. C., and Candel, S., Prog. Energy Combust. Sci. 26:225 (2000).

3. Katta, V. R., Carter, C. D., Fiechtner, G. J., Roquermore, W. M., Gord, J. R., and Rolon, J. C., Proc. Combust. Inst. 27:587 (1998).

4. Lee, J. C., Frouzakis, C. E., and Boulouchos, K., Combust. Sci. Technol. 158:365 (2000).

5. Santoro, V. S., Kyritsis, D. C., Liñán, A., and Gomez, A., Proc. Combust. Inst. 28:2109 (2000).

6. Kyritsis, D. C., Santoro, V. S., and Gomez, A., "Measurements and Computations of Scalar Dissipation Rate in Vortex Perturbed Flames," paper 72, Eastern States Meeting of the Combustion Institute, Hilton Head, SC, December 3-5, 2001. 
7. Santoro, V. S., Kyritsis, D. C., Smooke, M. D. and Gomez, A., "Nitric Oxide Formation during Flame/ Vortex Interaction," paper 1, Eastern States Meeting of the Combustion Institute, Hilton Head, SC, December 3-5, 2001.

8. Shiah, S. M., and Sichel, M., AIAA paper 93-0901.

9. Fichot, F., Harstad, K., and Bellan, J., Combust. Flame 98:5 (1994).

10. Raju, N., and Meiburg, E., Phys. Fluids 9:299 (1997).

11. Aggarwal, S. K., Park, T. W., and Katta, V. R., Combust. Sci. Technol., 113:429 (1996).

12. Santoro, V. S., Kyritsis, D. C., and Gomez, A., Proc. Combust. Inst. 28:1023 (2000).

13. Gao, L. P., D’Angelo, Y., Silverman, I., Gomez, A., and Smooke, M. D., Proc. Combust. Inst. 26:1739 (1996).

14. Massot, M., Kumar, M., Smooke, M. D., and Gomez, A., Proc. Combust. Inst. 27:1975 (1998).

15. Seshadri, K., Trevino, C., and Smooke, M. D., Combust. Flame 76:111 (1989).

16. Santoro, V. S., Liñán, A., and Gomez, A., Proc. Combust. Inst. 28:2039 (2000).
17. Santoro, V. S., Kyritsis, D. C., and Gomez, A., "Extinction Behavior of Either Gaseous or Spray Counterflow Diffusion Flames Interacting with a Laminar Toroidal Vortex," paper 159, Seventeenth International Colloquium on the Dynamics of Explosions and Reactive Systems, Heidelberg, Germany, July 25-30, 1999.

18. Gomez, A., and Chen, G., in Physical and Chemical Aspects of Combustion-A Tribute to Irvin Glassman (F. L. Dryer and R. F. Sawyer, eds.), Gordon and Breach, The Netherlands, 1997, pp. 461

19. Li, S. C., Libby, P. A., and Williams, F. A., Proc. Combust. Inst. 24:1503 (1992).

20. Greenberg, J. B., Cheatham, S., and Matalon, M., Combust. Sci. Technol. 131:277 (1998).

21. Greenberg, J. B., and Sarig N., Combust. Sci. Technol. 92:1 (1993).

22. Chen, G., and Gomez, A., Proc. Combust. Inst. 24:1531 (1992).

\section{COMMENTS}

Josette Bellan, Jet Propulsion Laboratory, USA. Can you please comment on the practical application of your results?

Author's Reply. The fundamental relevance of this study, we believe, is self-evident. Its practical relevance is indirect and can be best appreciated in the context of a flamelet description of turbulent flames. To the extent that practical spray flames can be modeled as an ensemble of laminar spray flamelets, studies of this kind are useful in guiding the development of turbulent combustion models to be applied to practical problems. 